\title{
Pénétration coloniale et résistance chez les Jarai Revisiter le rôle des colonisés dans la mise en place des frontières en Indochine.
}

Mathieu Guérin, Centre de recherche en histoire Quantitative (UMR 6583, université de Caen Basse-Normandie),

Jonathan Padwe, Departement of Anthropology and Environment Studies (université Yale)

La constitution de l'Indochine française est passée par la conquête du Vietnam, la mise sous protectorat du Cambodge, et la cession du Laos, mais aussi par l'intégration des hautes terres du centre de la péninsule. Celles-ci appartiennent à l'espace que James Scott a baptisé Zomia, une bande de terres d'altitude allant du Vietnam au Nord-Est de l'Inde à travers l'Asie du Sud-Est continentale et le Sud-Ouest de la Chine, qui constitue une zone de refuge pour les populations qui se maintiennent en dehors du contrôle des Etats ${ }^{1}$. Elles échappaient à toute administration avant l'arrivée des Français. Le processus d'assujettissement de ces territoires formant jusqu'alors une bulle entre Vietnam, Cambodge et Siam a dû passer par l'établissement de frontières ${ }^{2}$. La question s'est alors posée des critères, essentiellement naturels ou ethniques, pour tracer les frontières entre les différentes possessions françaises en Indochine. C'est ainsi que l'administration coloniale a dû fixer le sort des Jarai vivant dans les hautes terres du sud de l'Indochine, aux confins du Cambodge, de l'Annam et du Laos.

$\mathrm{Du}$ «nous sommes il est vrai chez les Djiaraïs, les pires voleurs entre les sauvages »du capitaine Cupet en 1893 dans Le Tour du Monde au «Les Jarai étaient forgerons, ils savaient tremper le fer et couler le cuivre (...) mais, pour les hommes, la guerre primait toute autre occupation » de Bernard Bourotte dans le Bulletin de la Société des Etudes Indochinoises en $1955^{3}$, les Jarai sont représentés pendant toute la période coloniale comme des gens particulièrement fourbes et violents, notamment après le meurtre de l'explorateur Prosper Odend'hal en 1904. Bien qu'ils soient, comme la grande majorité des habitants des hautes terres du sud de l'Indochine, avant tout des paysans essarteurs, des «paysans de la forêt » ${ }^{4}$, ils sont longtemps compris comme des guerriers féroces, comme si leur appartenance ethnique les prédisposait à la guerre. Il faut attendre les études du prêtre ethnologue Jacques Dournes dans le second $\mathrm{XX}^{\mathrm{e}}$ siècle pour sortir de cette caricature.

Nous chercherons à voir comment cette vision des Jarai a pu se construire et influer sur la politique menée envers eux par l'administration coloniale française, en étudiant la période correspondant au tracé des frontières dans les espaces où vivent les Jarai. Nous nous baserons essentiellement sur les archives administratives coloniales, ainsi que sur deux pièces d'archives privées particulièrement intéressantes : le journal de marche de Prosper Odend'hal et les mémoires du garde principal Pierre Dru ${ }^{5}$. Alors que la grande majorité des villages jarai ont été intégrés à l'Annam et dépendent aujourd'hui du Vietnam, on pourra s'intéresser tout particulièrement aux villages, qui bien que jarai, ont été rattachés au Cambodge. Nous insisterons sur les dynamiques qui ont mené au tracé des frontières en essayant de cerner le rôle que les populations colonisées ont pu jouer.

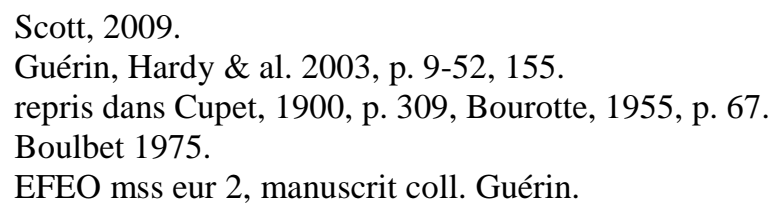




\section{Les Jarai, un groupe ethnique?}

D'abord compris comme un groupe ethnique, voire « une tribu », ceux que nous appelons les Jarai constituent un groupe parlant une langue austronésienne, le jarai, partageant des éléments d'une culture commune et un territoire ${ }^{6}$. Les Jarai, aujourd'hui environ 350000 personnes ${ }^{7}$, sont des habitants des hautes terres du centre de $1^{\prime}$ Indochine, vivant notamment dans la partie méridionale de la chaîne annamitique, dans les actuelles provinces de Kontum, Gia Lai et Dak Lak au Vietnam et de Ratanakiri au Cambodge, une zone située entre Cambodge, Laos, Annam et Cochinchine à la fin du XIX ${ }^{\mathrm{e}}$ siècle. Les Jarai partagent des traits culturels communs avec la plupart de leurs voisins des hautes terres. Ils pratiquent une religion animiste caractérisée par l'importance des sacrifices d'animaux domestiques et un calendrier rituel rythmant l'année agricole, afin de ne pas s'aliéner les esprits (yang) et les morts $($ atau $)$. Ils partagent une riche littérature orale ${ }^{8}$. La plupart pratiquent l'essartage, tout en étant souvent engagés dans d'autres systèmes de production, d'autres se tournent vers la riziculture humide inondée là où cela est possible, comme dans la vallée du Song $\mathrm{Ba}^{9}$.

Beaucoup d'observateurs ont dès les premiers contacts mis en doute la validité de la classification en tribus, rappelant que les Jarai, comme leurs voisins des hautes terres, n'ont pas d'organisation sociale au-delà de la famille, de la parenté ou du village. Le capitaine Cupet notait en 1893 :

«Aucun de leurs groupes ne rappelle par sa constitution sociale l'idée de tribu, telle que nous la comprenons chez les anciens ou chez certains populations nomades comme les Arabes ou les Indiens. Ce mot de tribu évoque un principe d'autorité sur l'ensemble du groupe et implique solidarité entre ses éléments. Chez les sauvages rien de pareil. » ${ }^{10}$

L'entité sociale et politique de base est alors le village et la communauté qui l'habite dans le cadre d'un finage connu. Au tournant du $\mathrm{XX}^{\mathrm{e}}$ siècle, les territoires jarai étaient difficiles à traverser et partiellement isolés des basses-terres, même s'ils étaient traversés par des voies commerciales les reliant de facto au reste des réseaux de l'Asie du Sud-Est. L'isolement dans lequel les différentes communautés villageoises vivaient a conduit à l'adoption d'ethnonymes en fonction des emplacements géographiques occupés par les autres communautés, que les ethnologues français du $\mathrm{XX}^{\mathrm{e}}$ siècle ont considérés comme des sous-groupes ethniques, étudiés notamment par Pierre-Bernard Lafont (figure 1) et Jacques Dournes (figure 2). Ainsi, les Jarai vivant le long de la moyenne Sésane, au confluent de cette rivière avec la Satay, ceux sur lesquels nous serons amenés à nous pencher davantage, sont appelés Jarai Cöbuan par Jacques Dournes. A la fin du XIXe siècle, il est possible, voire même probable, que cette zone géographique ait été occupée depuis peu par les Jarai.

Pour Jacques Dournes, l'identité jarai ne réside pas dans les coutumes, les occupations humaines ou la distribution géographique. Ainsi, les Jarai sont certes pour l'essentiel à l'époque de l'établissement de la domination coloniale française des paysans essarteurs guerriers, mais pas tous. Il a pu montrer que c'est à travers les mythes et notamment ceux qui entourent le système des Pötao que l'on peut saisir l'identité jarai. Les Khmers et les Vietnamiens, puis les Français les ont appelés « roi du feu » et « roi de l'eau ». Fraser en 1935 les qualifie de «rois des éléments » ${ }^{11}$. Pötao Ia, « le maître de l'eau » habite sur le versant

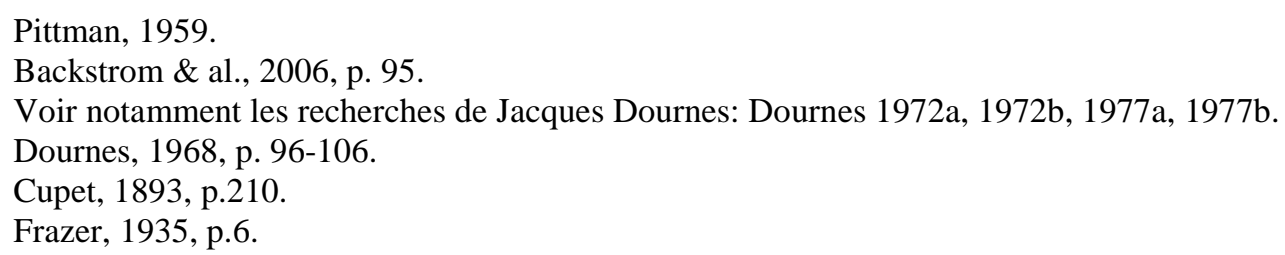


occidental du territoire jarai. Pötao Pui, «le maître du feu », et Pötao Angin, «le maître du vent », ce dernier longtemps ignoré des étrangers, habitent sur le versant oriental. Chaque Pötao représente l'expression d'une partie d'un tout, les trois éléments de la matière, le père, la mère, l'enfant, les éléments de la forge, etc., dispose d'un trésor palladium de son pouvoir, et possède des pouvoirs surnaturels liés à sa proximité avec le divin. Ils sont le lien nécessaire entre les hommes et entre les hommes et le divin. Le peuple jarai peut être divisé en sept clans matrilinéaires rattachés aux huit familles mythiques des origines. Pour Dournes, ce sont les Pötao et H'bia, fille du Pötao et qui à ce titre incarne la femme, qui garantissent la cohésion du peuple jarai à travers le mythe ${ }^{12}$.

\section{Les « sauvages » jarai}

Dans les années 1850, le missionnaire Charles Emile Bouillevaux présentent les Jarai comme «les brigands Charai, sauvages très redoutés " ${ }^{13}$, même s'il ne les a jamais rencontrés. Les premiers Français à être entrés en contact avec des Jarai sont les prêtres catholiques des Missions étrangères de Paris Jean-Claude Miche et Pierre Duclos qui fuyaient les persécutions au Vietnam en $1842^{14}$. Il faut attendre la fondation de la célèbre mission de Kontum, «la mission des sauvages $\gg{ }^{15}$ qui s'installent en 1849 chez les Bahnar, au nord des territoires jarai, pour que des Français vivent au contact des Jarai, des contacts qui sont loin d'être pacifiques ${ }^{16}$. La mission vit sur le qui-vive face aux raids de ses voisins du sud. La tension atteint son paroxysme lorsqu'en 1887-1888, après le pillage d'un convoi destiné à ravitailler la mission, le père Guerlach entreprend de mater les villages jarai de Plei Mea et Plei Yang. Il bat alors le rappel des villageois ayant à se plaindre d'eux et aurait ainsi rassemblé 1200 guerriers. Les groupes jarai sont battus et font la paix avec les missionnaires, même si ceux-ci continuent à être considérés comme des ennemis potentiels ${ }^{17}$.

L'ethnohistorien Oscar Salemink a pu montrer l'importance des missionnaires dans la construction des savoirs sur les habitants des hautes terres et ainsi sur la manière dont les décideurs de l'administration coloniale se les représentaient ${ }^{18}$. Ainsi l'utilisation des qualificatifs de «sauvages », «brigands », «pillards » pour les désigner vient d'abord des missionnaires. Ils sont repris ensuite dans les documents administratifs.

\section{Rivalité franco-siamoise pour le contrôle des hautes terres}

Les raids des Jarai sur la mission de Kontum à la fin du $\mathrm{XIX}^{\mathrm{e}}$ siècle sont à mettre en relation avec les avancées exogènes dans la région. Les années 1880 sont celles où les Siamois s'engagent dans la cordillère annamitique pour y asseoir leur domination, au moins nominale $^{19}$. Le village de Veunsai sur la Sésane est ainsi créé par le Chau Tham Phuoy, membre et dignitaire de la maison royale de Champassak alors sous suzeraineté siamoise, pour servir de base afin d'administrer les Brao, les Tampuon, les Jarai et les placer ainsi sous

\footnotetext{
$12 \quad$ Dournes, 1977a.

13 Bouillevaux, 1874, p.145.

14 Ils sont rapidement arrêtés par des mandarins vietnamiens et envoyés à Phu Yen, Dournes, 1977a, p. $15-16$.

15 Dourisboure, 1873

16 Dournes, 1977a, p.18-20 ; Maitre, 1912, 519-520.

17 Rapport sur l'action politique exercée par la mission de Kontum chez les Djaraïs du commis des services civils faisant fonction de commissaire à Attopeu Dulac au résident supérieur du Laos, 15 juillet 1904, arch. nat. outre-mer, ggi 5934 ; Maitre, 1912, p. 215, 523.

18 Salemink, 2003, p. 31-35.

19 Wyatt, 2002, p. 201-203; Maitre, 1912, p. 398-399, 521-523.
} 
l'orbite du Siam ${ }^{20}$. En face, à partir de l'Annam, de la Cochinchine et du Cambodge, les Français s'engagent de leur côté dans les hautes terres. En 1888-1889, un aventurier qui se fait appeler baron Marie de Mayrena, crée une confédération bahnar-sedang dont il devient le souverain, avec l'aide de la mission de Kontum. Désavoué, il ne peut rentrer en Indochine après un séjour en Europe où il a distribué les concessions et les titres nobiliaires de «son royaume $»^{21}$.

Les années 1888-1895 sont celles de la célèbre mission Pavie, lorsqu'une quarantaine d'explorateurs parcourent la cordillère annamitique sous la direction d'Auguste Pavie. Ils tracent plus de $35000 \mathrm{~km}$ d'itinéraires à pied, en bateau, à cheval ou sur éléphants, dressent des cartes, relève la géographie humaine et physique des espaces qu'ils traversent, permettant à la France de mieux connaître ces territoires sur lesquels elle commence à avoir des vues. Parmi les membres de la mission Pavie, deux surtout ont affaire aux Jarai : le capitaine Cupet et le lieutenant Dugast. La première rencontre entre Cupet et les Jarai est, d'après le récit qu'en fait Cupet, tendue. Il insiste sur les conflits entre les Jarai et le père Guerlach, leur propension à lancer des raids contre d'autres villages ou à chercher à le tromper ${ }^{22}$. Une grande partie de son récit d'exploration est centrée sur un incident symptomatique de la poussée simultanée des deux impérialisme siamois et français dans les hautes terres. Cupet arrête une colonne dirigée par des Siamois et des Laotiens de Champassak cherchant à faire reconnaître l'autorité siamoise sur les villages de la haute Srépok autour de Ban Don, à la limite des territoires jarai, et la pousse à faire demi-tour ${ }^{23}$.

En 1893, deux ans après la mission du capitaine Pavie, la France prend prétexte du suicide de son consul à Luang Prabang et de l'expulsion de deux commerçants français pour intervenir militairement au Laos ${ }^{24}$. Des canonnières sont envoyées devant le palais royal à Bangkok. Au nom de droits de la cour vietnamienne, largement hypothétiques, sur l'arrière-pays laotien, la France exige et obtient par le traité franco-siamois du 3 octobre 1893, la rive gauche du Mékong. Les territoires de la chaîne annamitique disputés entre Siamois et Français dépendent désormais de l'administration française.

Dans un premier temps, ils sont placés sous l'autorité du lieutenant-gouverneur de Cochinchine. Auguste Pavie installe les différents administrateurs du Laos français. Dans le sud, c'est le commandant Tournier qui prend en charge la gestion administrative et militaire des provinces de la rive gauche. L'année suivante, par arrêté du $1^{\text {er }}$ juin 1895 , le Laos est divisé en deux circonscriptions : le Haut-Laos avec pour capitale Luang-Prabang et le BasLaos, dont le commandant supérieur est le commandant Tournier qui siége à Khône. Le plateau du Dak Lak et les territoires jarai se trouvent alors dans le ressort du commissariat de Stung Treng, lui-même intégré au Bas-Laos ${ }^{25}$.

Pour autant, l'autorité française dans l'arrière-pays nouvellement acquis est alors largement fictive. Les Siamois ont abandonné à la France des territoires qu'ils ne contrôlaient pas euxmêmes. Le capitaine Cupet insiste sur le fait que les villages jarai ne paient en 1891 aucun

$20 \quad$ Guérin, 2008, p. 195, Baird 2009 p. 36.

$21 \quad$ Soulié, 1927 ; Salemink, 2003, 51-54.

$22 \quad$ Il est à peu près certain que Cupet a tendance à exagérer les réticences des villageois à l'aider dans ses explorations (Guérin, 2008, p. 32). Il ne semble pas que les Jarai, à aucun moment, ne l'aient empêché de mener à bien sa mission. Toutefois, pour les Français, c'est son récit qui fait foi et qui construit les représentations. Cupet, 1900, p. 310-311, 361, 370.

23 Cupet, 1900, p. 358-391; arch. nat. outre-mer ggi 9285.

24 Le Boulanger, 1931, p. 304-307.

25 Maitre, 1912, p. 536, Arch. nat. outre-mer, fm sg indo nf 616. 
tribut recognitif de la soumission aux Siamois ou aux Laotiens. Il mentionne néanmoins la présence d'un poste laotien à Plei Pi sur les hauteurs de Bokham ${ }^{26}$. De fait, il décrit un pays de frontière, dans l'acception de Turner du terme. Siamois et Laotiens sont présents, en contacts avec les habitants, mais ne semblent pas encore les avoir soumis à leur autorité. Les seuls qui semblent percevoir des tributs des villages de la région sont les Pötao ${ }^{27}$.

\section{Les premières tentatives pour soumettre les Pötao à l'autorité française}

Après l'installation des premiers administrateurs français à Stung Treng en 1894, la puissance coloniale cherche à reconnaître les villages jarai et à obtenir leur soumission. Le commissaire de Stung Treng Frébault accompagné d'une suite de près de quatre-vingt personnes dont dixsept miliciens et un garde principal se rend au village de Pötao Ia, mais doit rebrousser chemin précipitamment face à la résistance des habitants. Trois ans plus tard une nouvelle expédition est menée par le commissaire Castanier. Il s'agit notamment de mettre un terme à la traite des esclaves à laquelle participent les Jarai dans cette zone ${ }^{28}$. Disposant d'une force de cinquante miliciens commandés par deux gardes principaux français et accompagnée par le Chau Tham Phuoy ${ }^{29}$, elle se heurte encore une fois à la résistance des Pötao. 1500 guerriers attaquent la colonne qui réplique en détruisant le village de Pötao Ia, mais sans que ce dernier ne se rende ${ }^{30}$.

L'insistance de Tournier, commandant supérieur du Bas-Laos et donc supérieur hiérarchique direct de Frébault et Castanier, à vouloir s'imposer aux Pötao s'explique par la vision erronée qu'il a de la fonction de Pötao:

«Le village et le groupement d'un certain nombre de villages sous la direction plutôt paternelle d'un chef choisi est la seule et très élastique organisation politique de la plupart de ces Khas. Cependant chez les Djiaraïs on rencontre deux véritables rois ; l'un porte le titre de Roi du Feu et l'autre de Roi de l'Eau. Ils exercent de plus un véritable pontificat, car tous les Khas de l'extrême Sud les reconnaissent comme chefs religieux et leur envoient à ce titre un tribut, même ceux qui sont soumis à l'influence des missionnaires de Kon-Toum. » ${ }^{31}$

Il espère ainsi pouvoir dominer tout le «peuple» jarai en obtenant la soumission des Pötao. Tournier ne comprend pas la nature des Pötao. Ils sont un ciment de l'identité jarai, ils possèdent des pouvoirs surnaturels reconnus dans toute la péninsule, bénéficient d'une très grande influence, mais n'ont aucun pouvoir de commandement temporel sur les villages jarai $^{32}$, chaque village étant une entité socio-politique indépendante.

Face à l'incapacité de l'administration française de se faire entendre dans la haute Srépok, un commissariat au Darlac, dont le siège est d'abord à Ban Don avant d'être transféré à Ban Mé

$26 \quad$ Cupet, 1900, 308, 363, 367 et carte PL XV.

$27 \quad$ Le commissaire de Stung Treng Frébault expose dans un courrier au commandant supérieur du Bas Laos en 1896 que «le Chau-Tam visita d'abord successivement Yao-Net, Yao Lule, Yao Keng, Kassung, ChauYaï et Chau Noï, ces six villages paient tribut aux Sadètes », Arch. nat. Outre-Mer, ggi 21419. Prosper Odend'hal rapporte dans son journal de marche de 1904 plusieurs témoignages de villageois jarai et même du village mnong-rhadé de Ban Don payant un tribut aux Pötao avant l'arrivée des Français. EFEO mss eur 2, p. 135.

28 Lettre du commissaire du gouvernement à Stung Treng au commandant supérieur du Bas-Laos, 4 mars 1897, arch. nat. outre-mer ggi 20676.

29 Dès l'installation des Français, le Chau Tham se met à leur service. Il apporte ainsi son aide à l'expédition de 1894 vers Bokham. Coussot et Ruel, 1898, p. 109, 137-139, 162-163.

$30 \quad$ Rapport du commissaire de Stung Treng, avril-mai 1904, arch. nat. outre-mer rsl F2 ; arch. nat outremer ggi 20676 ; Maitre, 1912, p. 537.

31 Lieutenant-colonel Tournier, « Notice sur le Laos français », Revue Indo-Chinoise, 1900, p. 111-112.

32 Dournes, 1977a, p. 323-324. 
Thuot, est créé en 1899. L'ensemble de l'organisation administrative du Laos est revue : les deux Laos étant réunis dans une résidence supérieure unique confiée à Tournier, passé lieutenant-colonel ${ }^{33}$.

\section{La soumission des Jarai de la moyenne Sésane}

Les villages de la moyenne Sésane, comme ceux sous l'influence directe des Pötao, sont toujours indépendants et n'acceptent aucune domination. Ils sont pourtant épisodiquement en contact avec les Français. Après le passage de Cupet en 1891, ils reçoivent en 1894 la visite d'un groupe d'aventuriers français, Chabannes, Ruel, Voitel, Coussot, Pelletier, Lefebvre, venus tenter l'expérience de l'exploitation des mines d'or pour le compte de la Société française d'étude des mines de T'boc à Bokham. Les Français expliquent avoir reçu un « excellent accueil de ces Tiaraïs ». Un an après le traité franco-siamois de 1893, ce sont eux qui apprennent aux Jarai que leurs territoires dépendent désormais de la France ${ }^{34}$. L'expérience tourne au fiasco. Les «fièvres » et un gisement insuffisant viennent à bout des velléités des colons européens ${ }^{35}$.

Ce n'est qu'en 1902 que le commissaire du gouvernement à Stung Treng annonce que la soumission des Jarai de la moyenne Sésane a été obtenue. Dans son rapport de février 1903, il annonce que «d'après les renseignements qui viennent de m'être adressés par le Tiao Tham on peut considérer comme définitive la soumission de tous les villages djaraïs ${ }^{36}$. Les négociations ont été menées par le Chau Tham Phouy, ce qui permet à ce dignitaire laotien d'accroître son influence dans les hautes terres en augmentant sa clientèle. Les Jarai sont désormais soumis à l'impôt. Selon le régime alors en vigueur au Laos, ils doivent 1 \$ par homme valide au titre de la capitation, payable en numéraire ou en nature, et dix jours de prestations sur les routes ${ }^{37}$. Dans la mesure où leurs territoires sont éloignés des centres de commandement et où aucun projet de route n'apparaît dans les archives, il est probable que seule un impôt en nature au titre de la capitation leur est demandé sans que nous en connaissions la forme. Le Chau Tham charge un chinois installé à Bokham de faire rentrer l'impôt dû par les Jarai. Dans son rapport de juin, le commissaire de Stung Treng annonce triomphant :

«La récente soumission des villages djaraïs a définitivement assuré la tranquillité dans toute la province et les populations, reconnaissant l'efficacité de notre protection, sont animées aujourd'hui du meilleur esprit à notre égard. ${ }^{38}$

Il doit vite déchanter. Dès le mois de juillet, puis de nouveau en février-mars 1904, son collègue d'Attopeu lui signale que des villages de sa circonscription a été menacés par les Jarai dépendant de Stung Treng. Le commissaire de Stung Treng constate alors qu'il ne sait en fait pas grand chose de ce qui se passe dans l'arrière-pays de sa province. Une tournée d'inspection avec rencontre des notables du village jarai mis en cause semble ramener le calme, même si les Jarai demeurent très réticents à payer l'impôt recognitif de leur soumission $^{39}$.

\footnotetext{
33 Maitre, 1912, p. 541. Le Boulangier, 1931, p. 338-341.

$34 \quad$ Coussot et Ruel, 1898, p. 74, p. 137.

35 Coussot et Ruel, 1898 ; Maitre, 1912, p. 251-252.

$36 \quad$ Rapports du commissaire du gouvernement à Stung Treng, novembre-décembre 1902, janvier-février 1903, arch. nat. outre-mer, rsl F1.

$37 \quad$ Tournier, 1900, p. 127.

38 Rapport du commissaire du gouvernement à Stung Treng, mai-juin 1903, arch. nat. outre-mer rsl F1.

39 Rapport du commissaire de Stung Treng, avril-mai 1904, arch. nat. outre-mer rsl F2.
} 


\section{Le meurtre d'Odend'hal : un tournant}

Prosper Odend'hal est un homme atypique parmi les membres de l'administration française en Indochine. Il est né le 24 novembre 1867 à Brest où ses parents tiennent un commerce rue de Siam. Bachelier es lettres et diplômé de Saint-Cyr, parlant allemand et anglais, il commence sa carrière en Indochine comme sous-lieutenant au $4^{\mathrm{e}}$ régiment tonkinois. Perclus de dettes, il rejoint la garde indigène pour la solde en 1890. Il est alors affecté à la mission Pavie, puis à des missions topographiques dans le Binh Thuan, le Khanh Hoa et le Thua Thin. En 1894, nommé chancelier de résidence, il fonde le poste de Savannakhet au Laos. Odend'hal professe alors un certain mépris pour les habitants des hautes terres. Il écrit ainsi à son supérieur hiérarchique :

« sans doute cet hiver nous pourrons aller brûler 2 ou 3 villages, tuer quelques cochons et briser quelques jarres, mais le plus sûr moyen est de lancer le laotien sur ces gens-là. Quelques innocents pourront peut-être payer pour les coupables, mais bast ! Tous les Kha ne valent pas cher. $»^{40}$

Il n'en reste pas moins un fonctionnaire particulièrement apprécié. En 1895, le commandant supérieur du Bas-Laos Tournier écrit de lui : «Fonctionnaire des plus brillants : actif, instruit, ne ménageant ni sa peine ni sa santé ». Il participe aussi aux travaux de repérage du sanatorium du Lang Bian. Devenu vice-résident, il prend la direction de la province de Phan Rang. En 1903, il rentre en congés en France et en profite pour suivre les cours de sanskrit de Sylvain Levy ${ }^{41}$.

Il semble que son rapport aux habitants évolue, peut-être sous l'influence de sa femme laotienne. On lui reconnaît alors une forte empathie pour les habitants. Dans les jours qui précèdent son assassinat, il soigne pendant plusieurs jours une petite fille atteinte d'une très forte fièvre ${ }^{42}$. Jean Ajalbert explique ainsi dans un entretien au journal Action :

« lui, c'était l'annamitophile, le laophile, dans toute l'acceptation des termes. Il avait gardé de la perte d'une femme indigène la plus grande tristesse, que peu de ses amis ont soupçonnée sous ses airs de fête à Paris. Il avait d'elle un enfant ${ }^{43}$ élevé ici. $»^{44}$

Par ses nombreuses explorations et son poste à Savannakhet, il connaît bien les hautes terres et leurs habitants. C'est ainsi qu'à son retour de France, il demande et obtient une mission d'exploration des hauts plateaux au nom de l'Ecole française d'Extrême-Orient, comme l'explique le gouverneur général de l'Indochine Paul Beau au ministre des Colonies :

«M. Odend'hal, Administrateur de $3^{\mathrm{e}}$ classe des services civils, avait été dans les premiers jours de cette année, et sur sa demande, chargé par le Gouvernement Général d'une mission d'études linguistiques, ethnographiques et archéologiques dans la partie orientale du moyen Laos. Il était pleinement qualifié pour la mener à bien. » ${ }^{4}$

C'est dans ce cadre, qu'Odend'hal se rend d'abord à Dalat dans le massif du Lang Bian en février 1904. Sur la route, il s'arrête au poste de Dran. Le garde principal Pierre Dru qui commande le poste l'accompagne à la recherche des trésors des rois chams gardés par les

40 Rapport politique du chef du poste administratif de Savannakhet au résident supérieur en Annam, 12 juin 1895, arch. nat. outre-mer, ggi 20279.

41 Dossier personnel de Prosper Odend'hal, arch. nat. outre-mer ggi 30630, dossier des archives ministérielles sur le meurtre d'Odend'hal, arch. nat. outre-mer fonds ministériel sg indo 45, Louis Finot, «nécrologie de Prosper Odend'hal », Bulletin de l'Ecole française d'Extrême-Orient, tome 4, 1904, p. 529-537.

$42 \quad$ Journal de marche de Prosper Odend'hal, EFEO mss eur 2, p. 171-177.

43 Jean Georges Patrice né le 18 janvier 1898 à Hué, fils d'une femme laotienne nommée Monsati, reconnu à Phan Rang le 28 juin 1902 par Prosper Odend'hal.

$44 \quad$ Coupure de presse, arch. nat. outre-mer fonds ministériel sg indo 45.

45 Lettre du gouverneur général au ministre des colonies, 20 avril 1904, arch. nat. outre-mer fonds ministériel sg indo 45 . 
Churu :

«Monsieur Odendahl, homme charmant de parfaite éducation, sait fort bien inspirer confiance aux Moïs qui lui font voir des diadèmes et bracelets en or ciselés simplement déposés en pleine brousse dans des petites chapelles sur pilotis. ${ }^{46}$

Odend'hal estime pour sa part qu'il a «inventorié un dépôt de vieilleries chames ${ }^{47}$. Après Dalat et la station de Dankia, il pénètre en territoire rhadé et se rend au Dak Lak, le lac qui a donné son nom à la province du Darlac. Le 23 février, il est à Ban Mé Thuot où réside le commissaire français et un commerçant européen ${ }^{48}$. Il note que «l'autorité du commissaire du gouvernement ne paraît pas s'étendre très loin vers le nord ». Le 6 mars, il reprend la route et rejoint Ban Don sur la Srépok. Une semaine après, il atteint le premier village mixte jarairhadé, Plei Taly. Le 17 mars, il est au poste français de Cheo Reo ou Plei Chu sur le Song Ba qui, bien que sur le territoire du Laos, dépend administrativement de Song Cau en Annam. Il y recherche des informations pour entrer en contact avec les Pötao jarai, visite des vestiges chams et enlève des pierres portant des inscriptions. Il s'installe ensuite dans un petit village jarai dans l'espoir d'obtenir une entrevue avec Pötao Ia et Pötao Pui.

Depuis son entrée en territoire jarai, Odend'hal doit faire face à une sourde hostilité. « On sent que le pays est beaucoup moins ouvert et pénétré qu'au Dac Lak ${ }^{49}$. En mai-juin 1903, le capitaine Cottes s'était heurté à l'attitude belliqueuse des habitants. Ceux-ci sont encore totalement en-dehors du contrôle de la France. Le résident du Darlac, Besnard écrit alors :

«Malheureusement la circonscription est tellement étendue que ces derniers [villages insoumis] échapperont encore d'ici longtemps à notre influence, principalement dans la région des Khas Pis de la rive gauche de la Srépok et chez les Djaraïs au-delà de Ban Tali où les communications difficiles et l'esprit belliqueux des habitants s'opposent à toute pénétration pacifique. ${ }^{50}$

Pötao Ia accepte néanmoins de recevoir Odend'hal et de traiter avec lui le 31 mars. Le Pötao indique être prêt à reconnaître l'autorité des Français. Les négociations pour rencontrer le Pötao Pui sont beaucoup plus difficiles. Le 7 avril 1904, Odend'hal est invité à boire la jarre dans une maison du village de Plei Mé, où il pense recevoir la soumission du Pötao Pui. Il est alors entouré de Jarai qui l'attachent puis le tuent à coup de bûches, de sabres ou de lances avec les vietnamiens qui l'accompagnent.

Fonctionnaire d'élite, respectueux des habitants, Odend'hal est considéré comme la victime des «sorciers jarai ». Stenger, le chef du poste de Cheo Reo écrit ainsi :

«J'ai les larmes aux yeux quand je songe qu'un Monsieur qui a passé ici une quinzaine d'années comme officier, comme Inspecteur de la Garde Indigène, comme Commissaire au Laos, comme Résident et qui croit aux sentiments humains des Moïs, mais surtout des Djarraïs, que je lui ai dépeint dans toute leur fourberie, leur sauvagerie. Il me répondait toujours « Stenger, vous exagérez. » ${ }^{51}$

Le Pötao Pui Oï At est d'abord considéré comme le principal responsable de l'assassinat. Parmi les motifs invoqués pour expliquer la mort de l'explorateur, on rapporte qu'il a refusé la jarre et un morceau de poulet sacrifié pour sa venue. On soupçonne les Jarai de l'avoir tué en raison de son insistance à voir le sabre palladium de Pötao Pui, ce que Cupet avait déjà essayé de faire en 1891 sans succès. Dans son journal de marche, Odend'hal signale par

$46 \quad$ Mémoires de Pierre Dru, Guérin et Seveau, 2009, p. 180.

$47 \quad$ Lettre de Prosper Odend'hal à son ami Destenay, 14 février 1904, arch. nat. outre-mer ggi 17182. Finot, 1904, p. 534.

48 Bardin, commissaire du gouvernement et Lefèvre, agent de Pierre Sauvaire, marquis de Barthélémy.

49 Journal de marche de Prosper Odend'hal, EFEO mss eur 2, p. 141.

50 Rapport mensuel du commissaire au Darlac 16 avril-15 mai 1903, arch. nat. outre-mer rsl F1.

$51 \quad$ Lettre de Stenger à Vincilioni, 11 avril 1904, arch. nat. outre-mer 17182. 
ailleurs un ensemble des signes susceptibles d'être interprétés contre lui par les Jarai. Ainsi, le jour de son arrivée à Cheo Reo, le 17 mars, est marqué par une éclipse partielle du soleil. Il est de plus conscient du mécontentement entraîné par le fait qu'il a déplacé des pierres portant des inscriptions chames, considérées comme des pierres génies par les habitants, au point que dans sa rencontre avec Pötao Ia, il se sent obligé d'indiquer que les pierres ont été restituées à des villages des environs ${ }^{52}$. Autant de motifs qui accréditent la «sauvagerie » des Jarai, incapables de comprendre l'intérêt d'une mission scientifique ou agissant sous l'emprise de leurs superstitions.

L'enquête menée a posteriori et les dernières pages du journal de marche de Prosper Odend'hal accréditent une toute autre thèse. Ainsi, le commissaire d'Attopeu Dulac rappelle après une tournée sur place les rapports conflictuels entre les Jarai et les Français, mission catholique de Kontum, colons et administration ${ }^{53}$. Le père Guerlach avait mis à l'amende plusieurs villages jarai. Surtout, trois ans avant la venue d'Odend'hal, le colon français Céloron de Blainville ${ }^{54}$ avait envoyé des cadeaux à Pötao Pui pour lui acheter un cheval. Ne trouvant pas le cheval suffisamment beau au regard des cadeaux, il a demandé le recours de la force publique pour se saisir du Pötao. Le garde principal Stenger commandant le poste de Cheo Reo avait à cette occasion attaqué et incendié le village de Plei Mé et tué plusieurs personnes $^{55}$. Odend'hal était parfaitement au courant de ces tensions. Il écrit ainsi à son ami Destenay «j' aurais du mal à entrer en relations avec eux ; les Français s'étant jusqu'ici bornés à leur flanquer des coups de fusil » ${ }^{56}$, et dans son journal de marche à la date du 17 mars :

"Quant à la pénétration chez les Sadètes, Stenger me dit qu'il y a été une seule fois et que n'ayant pu entrer dans le village, il a attendu $1 \mathrm{j} 1 \frac{1}{2}$ à la porte. Campé avec ses hommes. Il avait été chargé par de Blainville d'aller arrêter le Sadète Loeung. Il avait donné un délai, $9 \mathrm{~h}$ du matin et cette limite ne recevant pas de réponse, il a pénétré dans le village. Très misérable paraît-il et entouré d'une enceinte de pierre. Les habitants en armes tout autour. Feux de salve. Tout le monde avait pris la brousse. $~^{57}$

Pour le prêtre ethnologue Jacques Dournes, il fait peu de doute qu'Odend'hal a été tué parce qu'il représentait une menace pour l'indépendance des Jarai ${ }^{58}$. On peut aller plus loin. Le garde principal Stenger a attaqué le village du Pötao Pui après que celui-ci a accepté les cadeaux envoyés par Céloron de Blainville. Or, pour les Jarai, l'échange des cadeaux et du cheval avec le Pötao relevait d'un échange créant un lien politique, et non d'une simple transaction commerciale, lien politique qui a été violemment brisé par les Français. L'arrivée d'Odend'hal avec un buffle en cadeau et suggérant une soumission n'avait rien pour les rassurer sur les intentions des Français.

\section{La réponse des Français à l'assassinat}

$52 \quad$ Maitre, 1909 , p. 307 ; 1912, p. 547 ; Cupet, 1900, 314 ; coupures de presse de 1904, arch. nat. outremer fonds ministériel sg indo 45 ; Journal de marche de Prosper Odend'hal, EFEO mss eur 2, p. 139, 161, 167.

53 rapport de Dulac, commis des services civils faisant fonction de commissaire du gouvernement à Attopeu au résident supérieur au Laos, 15 juillet 1904, arch. nat. outre-mer, ggi 5934.

54 Auteur d'une note sur «Les Moïs des régions du Song-Ba et du Darlac (Annam)», Revue de Géographie, 1903, p. 128-147, 229-253.

Rapport de l'inspecteur Vincilioni au résident supérieur en Annam, 9 juin 1904, Arch. nat. outre-mer 17182 ; rapport sur l'action politique exercée par la mission de Kontum chez les Djaraïs du commis des services civils faisant fonction de commissaire à Attopeu Dulac au résident supérieur du Laos, 15 juillet 1904, Arch. nat. outre-mer, ggi 5934 ; Lettre du résident de Plei Ku Derr Plantié au résident supérieur en Annam, 3 septembre 1906, arch. nat. outre-mer ggi 5939.

$56 \quad$ Lettre de Prosper Odend'hal à Destenay, 25 mars 1904, arch. nat. outre-mer 17182.

57 Journal de marche de Prosper Odend'hal, EFEO mss eur 2, p. 139-141.

$58 \quad$ Dournes, 1977a, p. 38 
Après l'annonce du meurtre d'Odend'hal, plusieurs colonnes de la garde indigène sont envoyées sur place : une dirigée par le chef du poste de Cheo Reo, Stenger, une autre par le résident du Darlac, Bardin et une troisième par l'inspecteur Vincilioni. Le gouverneur général Paul Beau donne à ce dernier des consignes de modération dans l'utilisation de la force :

« Je vous laisse juge des mesures à prendre pour saisir les assassins de M. Odend'hal. Mais il faut éviter de faire supporter à toute une population les conséquences d'un crime qui ne peut s'expliquer que comme une vengeance consécutive des violences antérieures au passage de notre malheureux agent. Nous devons éviter de semer de nouveau ferments de haine qui retarderaient encore la pénétration pacifique des tribus sauvages ${ }^{59}$

Vincilioni arrive au village du Pötao Pui le 8 mai. Il se rend alors avec une partie de son détachement à Plei Lut où il est attaqué selon ses dires par 1200 à 1500 guerriers jarai. Ces derniers laissent une cinquantaine de morts dans le combat. Le village est incendié. Dans un autre village, les paysans-guerriers jarai expliquent leur refus de la présence des Français :

«Ils m'ont fait répondre qu'ils avaient fait jadis soumission au Père Guerlach et après, ce dernier leur avait infligé amende vingt buffles au sujet église en paillottes, amende que moïs ont payé ; que dans ces conditions ils ne voulaient plus faire leur soumission et ne voulaient plus voir d'Européens chez eux. ${ }^{60}$

Vincilioni reste dans la région jusqu'en novembre 1904 et cherche à s'emparer, en vain, du Pötao Pui. Les villages doivent faire soumission, c'est-à-dire reconnaître l'autorité de l'administration coloniale et effectuer des prestations sur les routes, ou sont mis à l'amende. Les principaux responsables de la mort d'Odend'hal sont tués lors de l'engagement de Plei Lut ou exécutés sommairement dans le village du Pötao ${ }^{61}$. Plusieurs notables sont ensuite jugés en 1906 et condamnés à des peines de prison pour leur participation à l'assassinat. Le gouverneur général décide cependant de ne pas poursuivre le Pötao lui-même car il est potentiellement «un auxiliaire précieux pour [1']oeuvre de pénétration dans cette région difficile $»^{62}$.

Le meurtre d'Odend'hal a un retentissement et des conséquences importantes dans tout le pays jarai. Des troupes étrangères l'envahissent et l'occupent par la force, même si Vincilioni indique avoir limité l'utilisation de la violence. Tous les villages jarai sont tenus de reconnaître l'autorité française, même si les Pötao eux-mêmes échappent à cette humiliation. Pötao Pui et Pötao Ia doivent se cacher. Seul Pötao Angin, dont l'existence même ne semble pas connue des officiers français envoyés sur le terrain, n'est pas inquiété.

\section{La réaction des Jarai de la Sésane}

Pötao Pui, considéré par la plupart des administrateurs français comme un roi des Jarai, étant directement impliqué dans le meurtre d'Odend'hal, le commissaire de Stung Treng s'attend à des mouvements dans les villages jarai de sa juridiction. «les Khas Djaraïs plus ou moins soumis de la frontière » deviennent suspects.

«Le 18 avril, lorsque j'ai reçu le télégramme m'annonçant l'assassinat par les Sadètes du feu de M. Odend'hal, j'ai réuni les quatre autorités du chef-lieu et après avoir porté à leur connaissance cette triste nouvelle je leur ai donné l'ordre de faire surveiller les Djaraïs de la frontière pouvant être en relation avec les Sadètes de l'eau installés sur ce versant de la chaîne annamitique, non loin de ceux du feu

\footnotetext{
59 Lettre du gouverneur général au résident supérieur en Annam, 18 avril 1904, arch. nat. outre-mer 17182.

60 Télégramme de Vincilioni, 5 juin 1904, arch. nat. outre-mer ggi 17182.

$61 \quad$ Rapports de l'inspecteur Vincilioni 19 mai 1904, 19 juillet 190425 juillet 1904, 17 novembre 1904, arch. nat. outre-mer, ggi 5939.

62 Télégramme du gouverneur général au procureur général à saigon, 14 novembre 1905, arch. nat. outremer ggi 17182 .
} 
habitant de l'autre côté. » ${ }^{63}$

Il estime que Pötao Ia est susceptible de lever jusqu'à 2000 hommes dans 18 villages auxquels pourraient s'ajouter les Jarai de sa circonscription, nouvellement « soumis ».

« De l'avis de l'interprête Salgé et d'un Chinois, que j'ai pu voir, installé à Bo Kham depuis sept ans, il y aurait à craindre que les villages : de Ban Cha Tai qui peut disposer de 300 à 400 hommes, Ban Alé et Amé ensemble de 1 200, Ban Yao Vet de 100, Ban Yao Keng de 100, Ban Kassung de 800, Ban Kong Tek de 800 se joignent à eux. Les trois premiers de ces villages se trouvent dans le Darlac, les autres sur Stung Treng au milieu de la région occupée par les Khas insoumis. ${ }^{64}$

Les Français n'ont pas compris que si les Pötao ont effectivement la capacité théorique de rassembler de nombreux guerriers, cette mobilisation n'a rien d'automatique. Ils doivent obtenir le consentement de chacune des communautés villageoises. Or, les Jarai dépendant du Cambodge ne bougent pas. Aucun village ne rejoint le mouvement de résistance à l'occupation française. Ils se montrent juste, comme les années précédentes, réticents à payer l'impôt. Le commissaire de Stung Treng rapporte même qu'un colon français a pu se rendre chez eux.

«M. de Grenaud ${ }^{65}$ qui vient de parcourir le pays pour acheter des pirogues et est allé par ses propres moyens jusque dans les premiers villages Djaraïs, entre Lomphat sur la Srépok et Bokham sur la Sésane, a partout été bien accueilli à titre de commerçant. » ${ }^{66}$

\section{Tracer des frontières pour contrôler les populations}

Pour des raisons administratives particulièrement triviales, le meurtre d'Odend'hal pose immédiatement le problème du tracé exacte de la frontière. En effet, l'acte de décès d'Odend'hal doit mentionner le pays où il est mort ${ }^{67}$. Les fonctionnaires de l'Annam et du Laos se renvoient alors la balle. Chacun cherche à placer le lieu exact du meurtre chez le voisin pour dégager sa propre responsabilité tout en conservant le maximum de villages sous son autorité. Pour le gouverneur général Paul Beau, le meurtre de l'explorateur montre que les hauts plateaux du sud de l'Indochine, et notamment le pays jarai, sont insuffisamment contrôlés par la France en raison d'un maillage administratif trop lâche. Il accélère alors une consultation déjà engagée avant l'assassinat pour savoir à qui confier ces territoires.

Les fonctionnaires relevant de l'Annam insistent sur la rapidité des communications avec la côte et sur la présence de commerçants vietnamiens, les thuoc lai. Adhémard Leclère pour le Cambodge rappelle les liens commerciaux et politiques de longue date entre la vallée du Mékong et les hauts plateaux et la facilité des voies de communication ${ }^{68}$. Les administrateurs du Laos considèrent que les habitants n'accepteront aucune autre autorité et que leurs subordonnés lao refuseront d'être placés sous les ordres de dignitaires cambodgiens.

Dès le 20 avril 1904, le gouverneur général a arrêté sa décision. Il informe le ministre des colonies de sa volonté de modifier les frontières des circonscriptions de la zone en incorporant le Darlac à l'Annam, et ce, essentiellement pour des raisons de contrôle administratif du

\footnotetext{
63 Rapports du commissaire du gouvernement à Stung Treng, juin-juillet 1903, arch. nat. outre-mer, rsl F1, février-mars, mars-avril et avril-mai 1904, arch. nat. outre-mer, rsl F2.

$64 \quad$ Rapport du commissaire de Stung Treng, avril-mai 1904, Arch. nat. outre-mer rsl F2. Pötao Ia est celui qui vit le versant occidental de la montagne et donc le plus proche des Jarai de la Sésane.

Le comte de Grémaud : colon français installé dans l'île de Koh Logneu en face de Sambor, Guérin 2008 , p.179.

66 Rapport du commissaire de Stung Treng, aôut-septembre 1904, arch. nat. outre-mer rsl F2.

$67 \quad$ Correspondance, arch. nat. outre-mer 17182.

68 Guérin, 2003, p. 236-237.
} 
pays $^{69}$. Une commission du conseil supérieur de l'Indochine est saisie de la question et se prononce en faveur d'un rattachement à l'Annam, précisant que «le Cambodge désire que les Djaraï, les Radés \& les Cherraïs ${ }^{70}$ soient rattachés au Darlac et par conséquent à l'Annam. Sa Majesté le Roi du Cambodge partage entièrement cette manière de voir ${ }^{71}$. Ainsi, il est décidé à Hanoi, contre l'avis de la plupart des administrateurs de terrain de modifier en profondeur les frontières.

Suite à la convention franco-siamoise du 13 février 1904, les provinces de Mélou Prey et Tonlé Répou sur la rive droite du Mékong, face à Stung Treng, ont été incorporées au Cambodge. La France pense alors laisser au Cambodge le bourg de Stung Treng en fixant la frontière avec le Laos à la Srépok. Le roi Sisowath demande et obtient que le village khmer de Siempang sur la Sékhong soit aussi incorporé au Cambodge ce qui amène à intégrer la rive droite de la Srépok et la vallée de la basse Sésane. Par arrêté du gouverneur général du 22 novembre 1904, le Darlac est rattaché à l'Annam mais reste sous administration directe de la France et par arrêté du 6 décembre, Stung Treng est rattaché au Cambodge. La frontière entre Darlac (Annam) et Stung Treng (Cambodge) est fixée d'abord selon des critères physiques, sur la rivière Nam Thamm ${ }^{72}$.

Un nouvel arrêté vient modifier cette disposition l'année suivante. Le but est alors de regrouper les villages par groupes ethniques en «territoires de parcours et aires de dispersion des tribus », les paysans essarteurs étant perçus, à tort, comme nomades par nature ${ }^{73}$. Il y a certes eu de vastes mouvements de migrations dans les hautes terres dans la seconde moitié du $\mathrm{XIX}^{\mathrm{e}}$ siècle, mais on doit les considérer comme des conséquences de la très forte instabilité, des problèmes de sécurité et des épidémies à cette période et non des méthodes culturales des habitants. L'arrêté du 4 juillet 1905 prévoit ainsi que les Jarai devront répondre de l'Annam.

«Article $1^{\mathrm{er}}$.- La région montagneuse comprenant les territoires de parcours et aires de dispersion des tribus dénommées Sedang, Ha-Lang, Rognao, Ba-nar, Djaraïs et des sousgroupes de même famille, est réintégrée dans le territoire de l'Annam et constituée en une province autonome.

Art 2.- Le Chef-lieu administratif de cette circonscription sera jusqu'à nouvel ordre le village Djaraï de Pleikan-Derr. ${ }^{74}$

La recherche d'une homogénéité ethnolinguistique est sensée faciliter la tâche des administrateurs. Cela ne change pas grand chose pour les Jarai dépendant du poste de Cheo Reo, déjà placés sous autorité du chef de circonscription de Song Cau. Les villages situés autour des villages des Pötao qui étaient en dehors de la zone d'influence française et les villages jarai dépendant de Stung Treng se retrouvent théoriquement sous l'autorité directe des Français agissant au nom de l'Annam.

\section{Des frontières tracées sur les cartes à leur matérialisation}

La colonne Vincilioni et la fuite des Pötao ne s'accompagnent pas d'un retour au calme

\footnotetext{
69 Lettre du gouverneur général au ministre des colonies, 20 avril 1904, arch. nat. outre-mer fonds ministériel sg indo 45 .

70 Transcription du terme cambodgien pour désigner les Jarai.

Rapport de la $2^{\mathrm{e}}$ commission du Conseil supérieur sur les mutations territoriales en Annam, au Cambodge, en Cochinchine, et au Laos, sd [1904], arch. nat. outre-mer hcc 33.

$72 \quad$ Arch. nat. outre-mer hec 33 ; Forest, 1980, p. 167-180.

73 Guérin, 2009.

$74 \quad$ Plei Ku Derr. Arch. nat. outre-mer fonds ministériel sg indo nf 616.
} 
durable dans le pays jarai, comme escompté par les autorités françaises. Les troupes de la garde indigène doivent se maintenir dans des postes disséminés sur l'ensemble du territoire et faire face aux actes de rébellion.

En Annam, des commerçants et villageois vietnamiens sont attaqués. Pierre Dru qui dirige en 1907-1908 le poste de Plei Bang à la limite entre les territoires bahnar et jarai, raconte l'inquiétude qui accompagne le retour du Jarai Mlo arrêté après le meurtre d'Odend'hal puis acquitté. En mai 1906, des villages dépendant d'Attopeu au Laos sont attaqués et razziés par un groupe de guerriers venant d'une dizaine de villages différents menés par un Jarai de Ban Sop nommé Boum. Celui-ci est tué en cherchant à s'évader après son arrestation. Le même mois, un autre village Bok Nok sur la rive droite de la Sésane est attaqué, une vingtaine de personnes sont enlevées et vendues aux Jarai. Deux Jarai du village de Kong Lê Noi sont tués et un autre blessé à Moulapoumok en essayant de ramener chez eux des buffles volés. En juin, plusieurs villages dépendant d'Attopeu sont attaqués par des Jarai ${ }^{75}$.

En 1907-1909, une vaste opération de police est lancée chez les Jarai de l'Annam, avec colonnes de la garde indigène menaçant les villages afin d'obtenir la mise au pas des habitants. La violence règne alors en pays jarai. Le docteur Bouvaist qui organise une tournée de vaccine dans Moulapoumok en mai 1908 doit faire demi-tour à Ban Talao sur la Sésane sur les conseils du Chau Tham et des fonctionnaires cambodgiens de la province qui estiment « qu'il serait imprudent de s'aventurer trop près du territoire de l'Annam, les populations y étant surexcitées par les colonnes de police qui circulent au-delà de la frontière ${ }^{76}$. L'année suivante, le délégué de Veunsai écrit une lettre au ton inhabituellement dur pour ses collègues de l'autre côté de la frontière :

«Je m'étonne que ces peuplades guerrières ne se vengent pas plus souvent des exactions qu'elles sont contraintes de subir. Seule la terreur que leur inspirent les leçons que leur donne l'Annam, sous prétexte de vouloir les civiliser, peut l'expliquer. » ${ }^{77}$

Derrière les colonnes, s'engouffrent les missionnaires de Kontum. Deux missionnaires accompagnés d'une douzaine de catéchistes sont signalés à Yao Pamouk: «ils se sont emparés de tous les objets ayant trait au culte des génies en faveur chez les Khas et y ont mis le feu, répandant les cendres dans la forêt. les Djaraïs n'ont rien dit. ${ }^{78}$ À partir de 1909, le calme est rétabli, par la force ${ }^{79}$.

Ce contexte d'extrême violence orchestré par l'administration française en Annam explique en partie que des villages jarai aient cherché à sortir de son orbite. Le 22 juillet 1908, vingtdeux représentants de vingt-et-un hameaux de la moyenne Sésane ${ }^{80}$, disant rassembler cinq cents habitants, présentent un courrier commun adressé au Chau Tham Phouy.

«avons l'honneur de vous informer que nous étions chefs en tête de 500 habitants de race Khas Jiaraïs anciennement dépendant de la $\mathrm{P}^{\mathrm{ce}}$ de Moulapoumok (Veun Saï). Aujourd'hui nous avons appris que nos villages seraient attachés à l'Annam, alors nous nous disposons à nous séparer complètement de cette dépendance, car nous préférons dépendre à $\mathrm{M}^{\mathrm{rs}}$ l'Oknha Aranh Ruong Ruth et le conseiller Phouéï

75 Arch. nat. Cambodge rsc 25612, 25758, 26306; Maitre, 1912, p. 550 ; Mémoires de Pierre Dru, manuscrit, p. 165-170.

$76 \quad$ Lettre du délégué de Veunsai au résident de Stung Treng, 3 mai 1908, Arch. nat. Cambodge rsc 25668.

77 Lettre du délégué de Veunsai au résident de Stung Treng, 19 mai 1909, Arch. nat. Cambodge, rsc 25774 .

78 Rapport économique de Moulapoumok, décembre 1907-janvier 1908, arch. nat. Cambodge rsc 15455.

$79 \quad$ Maitre, 1912, p. 203-204, 550.

80 Ban Apia, Apo Niai, Apo Noi, Bokham, Chong, Katang, Kong Katet, Kong Kom, Kong Le, Kong Loun, Kong Pa Done, Kong Passou, Kouy Pa O, Lamok, Lom Romie, Phi, Some Niai, Some Noi, Takok, Ten Apo, Ten Loumphou. 
(dit Chau Tham) de la $\mathrm{P}^{\mathrm{ce}}$ de Moulapoumok, attendu que nous avons toujours payé nos tribus [sic] et impôts depuis lors du temps de nos parents à cette province. ${ }^{81}$

Ils demandent ainsi expressément à être rattachés au Cambodge, ou plus exactement à dépendre du gouverneur de Moulapoumok et du Chau Tham Phouy et menacent de quitter leurs villages et de prendre la forêt en cas de refus. Cinq autres villages font la même démarche en septembre 1909.

Les Jarai justifient leur requête par l'habitude ancestrale de payer l'impôt au Chau Tham « depuis lors du temps de nos parents », alors même que leur soumission remonte seulement à quelques années. Seuls trois des villages pétitionnaires figurent sur les listes des villages enregistrés aux rôles d'imposition de Stung Treng en 1901, Apia, Katang, Kong Pa Done ; la soumission des autres date très probablement de 1902 lorsque le Chau Tham Phouy avait négocié des soumissions dans la moyenne Sésane ${ }^{82}$. En fin de courrier, ils expliquent leur méconnaissance de la langue vietnamienne «qui pourra de telle sorte, nous rendre de grands embarras aux points de vues judiciaires ou des ordres de services ». Il est vrai qu'ils utilisent essentiellement le lao dans leurs relations avec l'administration.

La démarche de ses villages jarai et la forme qu'elle prend, une lettre écrite au Chau Tham Phouy qu'ils chargent de transmettre au résident supérieur du Cambodge, sont tout à fait surprenantes. Les villageois jarai sont illettrés. Ils signent la lettre avec leur empreinte digitale à Veunsai, au chef-lieu administratif, alors même que l'on sait que des Lao vivent dans leurs villages, notamment à Bokham, et auraient pu leur écrire la lettre s'ils avaient voulu passer par l'écrit. On peut supposer qu'une délégation de villageois représentant ces villages est venue à Veunsai rencontrer leur principal interlocuteur, celui qui a négocié leur soumission, le Chau Tham Phouy. Il est même probable que le village de Ban Apia, aussi appelé Plei Blia, situé à proximité immédiate de Ban Talao, en amont sur la rive droite de la Sésane, a joué un rôle d'intermédiaire en amenant la délégation devant le Chau Tham. Apia dépend déjà du Cambodge. Il apparaît sur la carte de la nouvelle circonscription de Stung Treng, et c'est le seul des villages pétitionnaires à figurer dans la liste des villages dépendant de Stung Treng en 1907. En 1909, lors de la deuxième vague de demande de rattachement au Cambodge, les cinq villages pétitionnaires sont représentés par un vieil homme de 65 ans, Meun Lat de Ban Apia $^{83}$. Le simple fait qu'une délégation regroupant des représentants de plusieurs villages se présente au chef-lieu indique que des palabres ont eu lieu entre villages avant l'envoi de celleci, mais nous n'en connaissons pas la teneur.

Il est tout à fait possible que le Chau Tham Phouy ait fait écrire, voire qu'il ait écrit lui-même, la lettre qui lui était adressée. En 1906, il avait été le premier gouverneur de la province de Moulapoumok. Un an plus tard, il est démis de ses fonctions, accusé de concussion et d'abus d'autorité, même s'il reste conseiller de la province. L'arrivée des délégations jarai est pour lui l'occasion de pouvoir se présenter comme un auxiliaire précieux pour les Français. Cela fonctionne parfaitement. En novembre 1909, lorsque la deuxième vague de villages jarai se présente, le résident de Stung Treng écrit au résident supérieur du Cambodge :

«Le Chau Tham Phoui, descendant des rois de Bassac, jouit dans toute la région de Moulapoumok et même au-delà, d'une influence considérable qui s'exerce particulièrement sur les tribus Champhuon, Pralao et Djaraï; c'est le seul chef indigène dont ces peuplades sauvages n'aient jamais contesté

$81 \quad$ Traduction d'une lettre de 22 chefs de villages jarai au Chau Tham Phouy, 3 juillet 1908, arch. nat. Cambodge rsc 25774 ; voir aussi le rapport du résident de Stung Treng du $1^{\text {er }}$ septembre 1908, arch. nat. outremer rsc 389.

$82 \quad$ Arch. nat. Cambodge rsc 25652.

83 Arch. nat. Cambodge rsc 26305, 25774, arch. nat. outre-mer hec 33. 
l'autorité. Il nous a rendu de réels services et les soumissions que nous avons reçues depuis un an, celles qui se préparent en ce moment, sont dues en grande partie à son intervention personnelle. ${ }^{84}$

Pour les Jarai, rejoindre le Cambodge, c'est échapper au climat de violence, aux colonnes de la garde indigène et à un contrôle strict imposé par l'administration de l'Annam. La frontière est ainsi utilisée pour se mettre à l'abri des Français !

\section{Vivre avec la frontière,}

La frontière créée en 1904 entre Stung Treng au Cambodge d'une part et Binh Dinh et Darlac en Annam d'autre part pose le problème des relations familiales, amicales ou commerciales transfrontalières. Théoriquement pour passer la frontière, les habitants doivent être porteurs de laissez-passer délivrés par les autorités françaises à partir de postes qui peuvent être à plusieurs jours de marche des villages. À défaut, ils peuvent être arrêtés. Dès avril 1908, le résident supérieur au Cambodge et le résident supérieur en Annam se mettent d'accord sur ce point autour d'une suggestion du résident de Stung Treng: les habitants du Cambodge pourront se rendre au Darlac et jusqu' au Binh Thuan, sans avoir à demander de laissez-passer, simplement en étant porteur de leur carte d'imposition personnelle. Ces cartes n'existant pas en Annam, la réciproque n'est pas possible ${ }^{85}$.

Enfin, l'impôt, tel qu'il est exigé par l'administration du protectorat français au Cambodge et prélevé par le Chau Tham n'était pas très lourd, dans la mesure où l'un et l'autre pouvaient se montrer conciliant. Théoriquement, les Jarai qui rejoignent le Cambodge sont soumis à la capitation et à la corvée. Chaque homme valide doit $2 \$ 65$, auxquels s'ajoutent 10 cents destinés au mékhum, au titre de la capitation, et 10 jours de corvée qui peuvent être rachetés pour $3 \$$. Ils obtiennent immédiatement la possibilité de payer en nature: armes, sabres, arbalètes, arcs, flèches, boucliers, lames, pirogues, peaux, cornes, cardamomes, cire, poudre et pépite d'or, ramie, ivoire, cornes de rhinocéros, fiel d'ours, paddy, buffles, boeufs, chevaux, bois, rotins, bref tout ce que l'on trouve sur les marchés et qui sert aux échanges, est accepté. Par ailleurs, le Chau Tham comme l'administration française tolèrent une importante dissimulation fiscale. Les Français cherchent à être agréables aux Jarai.

La mise en place d'une frontière entre villages jarai de l'Annam et du Cambodge entraîne un certain nombre de contraintes pour les habitants, même si matériellement, la délimitation n'existe d'abord que sur les cartes du service géographique de l'Indochine. Les villageois doivent se munir de leur carte d'imposition pour aller visiter leurs amis, leur famille ou leurs partenaires commerciaux restés en Annam. Toutefois, ces cartes n'ayant pas de photos et ne comportant qu'une description sommaire du porteur, il suffit que quelques personnes dans le village paient l'impôt pour disposer de cartes qui servent ensuite à l'ensemble de la communauté. Le nombre ridiculement bas d'inscrits dans les rôles montrent que cette pratique est fréquente.

En matière de commerce transfrontalier, le seul qui soit réellement touché est celui des éléphants. Les taxes à l'exportation mises en place au Cambodge, puis pour l'ensemble de l'Indochine entraîne une chute des échanges ${ }^{86}$. Toutefois, les villages jarai sont probablement

\footnotetext{
${ }_{84}$ Lettre du résident de Stung Treng au résident supérieur, 8 novembre 1909, arch. nat. Cambodge rsc 25601 .

85 Correspondance entre le résident de Stung Treng et le résident supérieur au Cambodge, et entre le résident supérieur au Cambodge et le résident supérieur en Annam, arch. nat. du Cambodge, rsc 1019.

86 Guérin, 2001, p. 48-49.
} 
épargnés par cette conséquence de la mise en place de la frontière, les Jarai n'étant pas des capteurs d'éléphants et la contrebande à la frontière leur permet toujours de se procurer les pachydermes dont ils peuvent avoir besoin ${ }^{87}$.

\section{Les atermoiements français}

La démarche des Jarai visant à revoir le tracé de la frontière est singulière. On trouve peu de cas de contestation organisée des frontières dans les archives de l'Indochine française. La réponse que lui donne l'administration française est plus surprenante encore : elle accède à la demande des villageois. En octobre 1908, ils sont rattachés à la province de Moulapoumok, dépendant de la résidence de Stung Treng, et donc au Cambodge. Le résident de Stung Treng va même jusqu'à considérer que «ces villages n'ont jamais cessé d'appartenir au Cambodge » et que leur rattachement à l'Annam résulte d'une erreur de nom entre deux rivières, pour marquer la délimitation entre les deux pays. Ce faisant, il méconnaît totalement l'arrêté 4 juillet 1905 qui remplace les critères physiques de délimitation par des regroupements par ensembles ethnolinguistiques.

Il s'agit d'une première concession d'envergure de l'administration coloniale. Celle-ci ne s'intéresse que de manière très marginale aux desiderata des populations lorsqu'elle trace les frontières. Pierre Lamant montre que ces frontières sont avant tout définies de manière à favoriser le fonctionnement de l'administration coloniale ${ }^{88}$. À travers la décision prise transparaît certes la rivalité entre les différentes administrations, mais aussi et surtout, la volonté de ne pas mécontenter les Jarai, ou plus exactement, celle de saisir l'occasion de leur être agréable. Les Jarai apparaissent en effet comme des fauteurs de troubles potentiels qu'il convient de ne pas froisser, tout en les contrôlant.

Un poste de milice est créé à Bokham en juillet 1909, placé sous le commandement d'un balat $^{89}$, le fils du Chau Tham Phouy, Poui. Il vise dans l'esprit des administrateurs français autant à surveiller les nouveaux villages cambodgiens et à percevoir l'impôt qu'à les protéger des commerçants vietnamiens perçus comme des escrocs.

«Les villages ayant fait leur soumission, restent tranquilles ainsi qu'ils l'ont promis, mais puisqu'ils ont renoncé à se faire justice eux-mêmes, ils demandent à l'administration à laquelle ils paient l'impôt de les protéger. ${ }^{90}$

En mai 1910, le délégué français de Veunsai organise une tournée dans l'arrière-pays pendant laquelle il passe à Bokham. Les Jarai lui indiquent qu'ils acceptent de payer l'impôt, ce qui n'est pas toujours suivi dans les faits.

Le rattachement de ces villages au Cambodge ne va pas sans poser de problèmes avec la délégation de Kontum en Annam. Lorsque l'administrateur Klein est nommé résident de Veunsai en octobre 1910, il est chargé de régler «le conflit soulevé à propos des Djarais et des territoires de parcours de ces derniers, revendiqués par l'Annam ${ }^{91}$. Une mission de reconnaissance est alors confiée à l'explorateur Henri Maitre, à l'issue de laquelle onze

\footnotetext{
$87 \quad$ Arch. nat. Cambodge rsc 25758.

$88 \quad$ Pierre Lamant in Lafont, 1989, p. 173.

$89 \quad$ Adjoint d'un gouverneur de province au Cambodge.

90 Rapport annuel du résident de Stung Treng au résident supérieur au Cambodge, 1909, arch. nat. Cambodge 26268.

$91 \quad$ Rapport politique du résident de Stung Treng au résident supérieur au Cambodge, octobre 1910, arch. nat. outre-mer rsc 389.
} 
hameaux sont de nouveau rattachés à l'Annam, sans que cela ne crée de difficultés ${ }^{92}$. En 1910, les opérations de police en Annam sont terminées et le Cambodge a engagé à partir du poste de Bokham une tentative de reprise en main des populations locales, même si celle-ci s'avère peu convaincante. En avril 1911, le résident de Stung Treng s'insurge encore contre l'«état de paix armée dans lequel ils se trouvent vis-à-vis de l'administration cambodgienne $»^{93}$. Un an plus tard, la maison du balat de Bokham est incendiée.

\section{Résistance et habilité tactique des Jarai}

Malgré les observations des explorateurs, pionniers avant l'heure de l'ethnographie tel le capitaine Cupet, qui ont noté la diversité interne des différents groupes, comme les Jarai, et l'inadéquation des notions occidentales de groupe ethnique ou de tribu à leur situation, les politiques à l'égard des habitants des hautes terres restent pendant toute la période coloniale marquées du sceau de l'ethnicité ${ }^{94}$. L'émergence d'une représentation des Jarai comme un groupe ethnique homogène est la conséquence directe des mécanismes de construction du savoir colonial et des classifications ethniques. La mise en oeuvre des politiques ethniques subséquentes a eu des conséquences auxquelles les Etats contemporains de l'Asie du Sud-Est doivent encore aujourd'hui faire face. La démarcation des frontières entre les Etats constituant l'Indochine française en est un exemple frappant tant elles sont marquées par les efforts faits pour prendre en considération les classifications ethniques.

Dans les travaux historiques sur les hautes terres du sud de l'Indochine, les années 1904-1905 apparaissent comme celles d'une prise en compte politique de l'importance de cette région. Les auteurs décrivent les modifications territoriales comme des décisions politiques des autorités françaises et notamment du gouverneur général Paul Beau, donnant l'impression d'un ordonnancement géostratégique d'ensemble pour l'Indochine ${ }^{95}$. Certes celui-ci a existé et le début du $\mathrm{XX}^{\mathrm{e}}$ siècle chez les Jarai s'inscrit bien dans la troisième ère de l'évolution de l'humanité décrite par James Scott, celle pendant laquelle les périphéries à l'écart des Etats sont investies par ceux-ci ${ }^{96}$. La France souhaite au début du $\mathrm{XX}^{\mathrm{e}}$ siècle contrôler les hautes terres et des postes sont construits à cet effet.

Toutefois, lorsqu'on se penche à l'échelle du terrain, des villages, on est frappé par la capacité des Jarai, alors au coeur de ce processus, à instiller le rythme du changement. La représentation des Jarai comme une tribu de guerriers féroces et dangereux ${ }^{97}$, alors qu'ils vivent au coeur de la zone frontière entre Stung Treng, Attopeu, Kontum et le Darlac, empêche d'abord l'administration coloniale d'agir. Il faut le meurtre de Prosper Odend'hal, dans lequel est directement impliqué le Pötao Pui, pour que la France mette en oeuvre une répartition par pays des hautes terres. La capacité de résistance des Jarai et l'importance du Pötao, ou plutôt l'image que s'en font les Français, expliquent que le gouverneur général accepte d'attendre encore cinq ans pour obtenir une soumission formelle des villages, alors même que celle-ci est obtenue en déployant des moyens qui restent modestes - quelques

\footnotetext{
92 Arch. nat. outre-mer rsc 389 ; arch. nat. Cambodge rsc 26268 ; Maitre, 1912, p. 244-245.

93 Rapport politique du résident de Stung Treng au résident supérieur au Cambodge, avril 1911, arch. nat. outre-mer rsc 389

94 Salemink, 2003, p. 1-39; Goudineau, 2000.

95 Maitre, 1912, p. 547-548; Bourotte, 1955, p. 84-85,89; Forest, 1980, p. 167-180 ; Brocheux et Hémery, 2001, p. 88-89; Guérin, 2003, p. 235-237.

$96 \quad$ Scott, 2009, p. 324

$97 \quad$ Il est saisissant de lire sous la plume d'Henri Maitre lorsqu'il relate son séjour chez les Jarai de la Sésane: «De ce côté de la Sésan; au fond, tout ce monde a une peur bleue des pillards brao signalés dernièrement et répugne à jouer du coupe-coupe dans la forêt et la brousse », Maitre, 1912, p. 246.
} 
détachements de la garde indigène, ce qui relativise le danger effectif que représentaient les Jarai. Le Pötao lui-même évite les Assises alors même qu'il est impliqué dans l'assassinat d'un administrateur français.

Ce qui se passe ensuite avec les «Jarai du Cambodge» montre de manière très claire les dispositions de ceux-ci pour contourner les décisions de l'administration coloniale, voire imposer leur volonté. Avec le soutien actif du Chau Tham Phouy, l'arrêté du 4 juillet 1905 du gouverneur général de l'Indochine proposant une frontière par répartition ethnique est ignoré, parce que les Jarai de la moyenne Sésane n'en veulent pas. Ils remettent ainsi en cause la conception très occidentale d'une homogénéité et d'une cohérence sociale, voire politique, propre des groupes ethnolinguistiques. Les Jarai appartiennent tous aux sept clans matrilinéaires, ils reconnaissent les Pötao comme les garants de l'identité jarai, mais restent des paysans-guerriers dont la structure politique de base est la cité villageoise. Et ces cités se maintiennent en dehors du contrôle effectif de la France, même après qu'elles ont reconnu l'autorité de la France. Ils renégocient à plusieurs reprises l'impôt recognitif de la soumission, qui n'est perçu que lorsque les habitants daignent le verser. Les attaques de villages, l'esclavage, la traite même, l'essartage se maintiennent sans que les Français n'aient la possibilité d'aller à l'encontre de ces pratiques qu'ils condamnent ${ }^{98}$. Comme le cas des Jarai de la moyenne Sésane le montre, la pratique coloniale a parfois dû céder devant les réalités des politiques locales. À y regarder de plus près, si c'est bien la France qui impose le principe du découpage territorial et des frontières, la mise en oeuvre de la tutelle coloniale sur les Jarai apparaît aussi comme une suite de renoncements de la France, dont les conséquences sont encore perceptibles aujourd'hui. Une frontière internationale coupe le pays jarai, mais elle ne vaut que pour ce que les Jarai acceptent d'en faire.

\section{Bibliographie}

Backstrom, Maria, Ironside, Jeremy, PAterson, Gordon, PADwe, Jonathan, BAIRD, Ian, Indigenous Traditional Legal Systems and Conflicts Resolution in Ratanakiri and Mondulkiri Provinces, Cambodia, United Nations Development Programme, Cambodian Ministry of Justice Legal and Judicial Reform Programme, Phnom Penh, 2006,

http://regionalcentrebangkok.undp.or.th/practices/governance/a2j/docs/CaseStudy-03-

Cambodia.pdf.

Blainville, Céloron de, «Les Moïs des régions du Song-Ba et du Darlac (Annam) », Revue de Géographie, 1903, p. 128-147, 229-253.

BAIRD, Ian G., «From Champasak to Cambodia : Ya Chao Tham (Chao Thammatheva), a wily and influencial Ethnic Lao Leader », Aséanie, n²3, juin 2009, p. 31-62.

BouillevauX, R.P. Charles-Emile, L'Annam et le Cambodge. Voyages et notices historiques, Victor Palmé, Paris, 1874, 537 p.

Boulbet, Jean, Paysans de la forêt, EFEO, Paris, 1975, 150 p.

BourotTE, Bernard, «Essai d'histoire des populations montagnardes du sud-indochinois jusqu'à 1945 », Bulletin de la Société des Etudes Indochinoises, tome XXX, fasc. 1, Saigon, $1955,116 \mathrm{p}$.

Brocheux, Pierre, Hemery, Daniel, Indochine, la colonisation ambiguë 1858-1954, La

98

Guérin, 2008, p. 219-244. 
découverte, Paris, 2001, 451 p.

Coussot, Alfred, Ruel, Henri, Douze mois chez les sauvages du Laos, Challamel, Paris, 1898, $352 \mathrm{p}$.

CuPET, Pierre, Voyages au Laos et chez les sauvages du Sud-Est de l'Indochine, La mission Pavie, t.III, Ernest Leroux, Paris, 1900, 428 p.

DOURISBOURE, Pierre, Les sauvages ba-hnars (Cochichine orientale), souvenirs d'un missionnaire, E. de Soye, Paris, 1873, 449 p.

DouRnES, Jacques, «Bois-Bambou (köyau-ale). Aspect végétal de l'univers jörai », Journal d'agriculture tropicale et de botanique appliquée, $\mathrm{n}^{\circ} \mathrm{XV}-4-11,1968$, p. 89-164, 369-498.

Dournes, Jacques, La culture jörai, Musée National d'Histoire Naturelle, Paris, 1972a, $104 \mathrm{p}$.

DOURNES, Jacques, Coordonnées, structures Joraï familiales et sociales, Institut d'ethnologie, Paris, 1972b, $323 \mathrm{p}$.

Dournes, Jacques, Pötao : une théorie du pouvoir chez les Indochinois Jorä̈, Flammarion, Paris, 1977a, $365 \mathrm{p}$.

DouRnes, Jacques, Akhan : contes oraux de la forêt indochinoise, Payot, Paris, 1977b, 278 p.

FINOT, Louis, "nécrologie de Prosper Odend'hal », Bulletin de l'Ecole française d'ExtrêmeOrient, tome 4, 1904, p. 529-537.

FOREST, Alain, Le Cambodge et la colonisation française, histoire d'une colonisation sans heurts (1897-1920), L'Harmattan, Paris, 1980, 546 p.

FrAZER, James G, Le Roi magicien dans la société primitive, Geuthner, Paris, 1935, vol. II, 399 p.

Goudineau, Yves, «Ethnicité et déterritorialisation dans la péninsule indochinoise : considérations à partir du Laos », Autrepart, n¹4, 2000, p. 17-31.

GuERIN, Mathieu, Paysans de la forêt à l'époque coloniale : la pacification des habitants des hautes terres du Cambodge, Bibliothèque d'Histoire rurale, AHSR, Caen, 2008, iv+354 p.

Guerin, Mathieu, Seveau, Arnoult, «Aux côtés du tigre, sur les hauts plateaux de l'Annam. Les mémoires de Pierre Dru, garde principal sur la route du Lang Bian en 1904 », OutreMers, n³62-363, juin 2009, p. 155-192.

GuERIN, Mathieu, «Essartage et riziculture humide, complémentarité des écosystèmes agraires à Stung Treng au début du $\mathrm{XX}^{\mathrm{e}}$ siècle », Aséanie, nº 8, décembre 2001, p. 35-55

GuERIN, Mathieu, Des casques blancs sur le Plateau des Herbes. La pacification des aborigènes des hautes terres du sud de l'Indochine, 1859-1940, thèse d'histoire sous la direction d'Alain Forest, Université Paris 7-Denis Diderot, Paris, 2003, 516 p.

GUERIN, Mathieu, «L'administration coloniale française face à l'essartage en Indochine », in Philippe MAdELINE and Jean-Marc MoricEAu (ed.), Terres de conquêtes, terres de déprise. Enjeux fonciers, agricoles et cynégétiques, Enquêtes rurales, n¹2, 2009, p. 87-106.

Guerin, Mathieu, Hardy, Andrew, Tan Boon Hwee, Stan, Nguyen Van Chinh, Des montagnards aux minorités ethniques, quelle intégration nationale pour les habitants des hautes terres du Viêt Nam et du Cambodge ?, L'Harmattan-IRASEC, Paris-Bangkok, 2003, $354 \mathrm{p}$.

LAMANT, Pierre, «La frontière entre le Cambodge et le Viêt Nam du milieu du XIX ${ }^{\mathrm{e}}$ siècle à 
nos jours, in Pierre-Bernard Lafont, Frontières du Vietnam : histoire des frontières de la péninsule indochinoise, L'Harmattan, Paris, 1989, p. 156-182.

Le Boulanger, Paul, Histoire du Laos français. Essai d'une étude chronologique des principautés laotiennes, Plon, Paris, 1931, 383 p.

MAITRE, Henri, Les jungles mö̈, mission Henri Maître (1909-1911). Indochine sud-centrale, Exploration et histoire des hinterlands moï du Cambodge, de l'Annam, de la Cochinchine et du bas Laos, Larose, Paris, 1912, 580 p.

PItTMAn, S., «Jarai as a member of the Malayo-Polynesian Family of Languages in Asian Culture », Asian Culture, ${ }^{\circ} 1,1959$, p. 59-67.

SALEMINK, Oscar, The Ethnography of Vietnam's Central Highlanders, a historical contextualization, 1850-1990, University of Hawai'i Press, Honolulu, 2003, 383 p.

ScotT, James C., The Art of Nor Being Governed. An Anarchist History of Upland Southeast Asia, Yale University Press, New Haven-Londres, 2009, 442 p.

Soulie, Maurice, Marie $1^{\text {er }}$, roi des Sedang, 1888-1890, Marpon, Paris, 1927, 239 p.

TOURNIER, lieutenant-colonel, «Notice sur le Laos français », Revue Indo-Chinoise, 1900, $191 \mathrm{p}$.

WyATT, David, Thailand, a Short History, Silkworm books, Bangkok, 2002, 351 p. 